\title{
Anti-Saccharomyces cerevisiae mannan antibodies combined with antineutrophil cytoplasmic autoantibodies in inflammatory bowel disease: prevalence and diagnostic role
}

J-F Quinton, B Sendid, D Reumaux, P Duthilleul, A Cortot, B Grandbastien, G Charrier, $S$ R Targan, J-F Colombel, D Poulain

\begin{abstract}
Background-Perinuclear antineutrophil cytoplasmic autoantibodies (pANCA) are a well recognised marker for ulcerative colitis. Antibodies to oligomannosidic epitopes of the yeast Saccharomyces cerevisiae (ASCA) are a new marker associated with Crohn's disease.

Aims-To assess the value of detecting pANCA and/or ASCA for the diagnosis of ulcerative colitis and Crohn's disease.

Methods-Serum samples were obtained from 100 patients with Crohn's disease, 101 patients with ulcerative colitis, 27 patients with other miscellaneous diarrhoeal illnesses, and 163 healthy controls. Determination of PANCA and ASCA was performed using the standardised indirect immunofluorescence technique and an ELISA, respectively.

Results-The combination of a positive pANCA test and a negative ASCA test yielded a sensitivity, specificity, and positive predictive value of $57 \%, 97 \%$, and $\mathbf{9 2 . 5 \%}$ respectively for ulcerative colitis. The combination of a positive ASCA test and a negative pANCA test yielded a sensitivity, specificity, and positive predictive value of $49 \%, 97 \%$, and $96 \%$ respectively for Crohn's disease. Among patients with miscellaneous non-inflammatory bowel disorders, three were ASCA positive and two were pANCA positive. One control was ASCA positive. The presence of ASCA in patients with Crohn's disease was associated with small bowel involve-
\end{abstract} ment.

Conclusion-ASCA and pANCA are strongly associated with Crohn's disease and ulcerative colitis, respectively. Combination of both tests could help the diagnosis of inflammatory bowel disease. (Gut 1998;42:788-791)

Keywords: Crohn's disease; ulcerative colitis; antineutrophil cytoplasmic autoantibodies; anti-Saccharomyces cerevisiae mannan antibodies

Inflammatory bowel diseases (IBD) are subdivided into ulcerative colitis (UC) and Crohn's disease (CD). Several lines of evidence suggest that $\mathrm{CD}$ and UC are different diseases. However, some patients $(10-12 \%)$ cannot be easily classified into either and a final diagnosis of indeterminate colitis is made. ${ }^{12}$ Making an earlier, more accurate diagnosis of IBD is important as the management of CD and UC is different, especially when surgery is planned. A search for serological tests to differentiate CD from UC has been underway for a long time. An ideal serological marker should have high sensitivity, high specificity, and high predictive values.

A subset of antineutrophil antibodies, commonly referred to as perinuclear antineutrophil cytoplasmic autoantibodies (pANCA) has been reported in sera from patients with IBD. The prevalence of pANCA varies from $40 \%$ to $80 \%$ in UC and from $0 \%$ to $20 \%$ in $\mathrm{CD}^{3-8}$ Since 1988, systemic antibodies against the yeast Saccharomyces cerevisiae have been reported in sera from patients with CD. Although great variation was found both in patients' antibody responses and in the relative antigenicity of different strains, these antibodies have been associated with CD and not UC..$^{9-11}$ We recently showed that this serological response recognises mannose sequences in the cell wall mannan of $S$ cerevisiae strain Sul (formerly $S$ uvarum 1, a species now classified within $S$ cerevisiae). ${ }^{12}$ Using the crude mannan from this strain as an antigen in an enzyme linked immunosorbent assay (ELISA), we found that testing for the presence of anti- $S$ cerevisiae mannan antibodies (designated ASCA) was $64 \%$ sensitive and $77 \%$ specific for discriminating CD from UC and $89 \%$ specific for distinguishing CD from controls. ${ }^{12}$

In the present study, the association between pANCA and UC and ASCA and CD was evaluated by single or combined use of these tests. The relation between serological test results and clinical parameters of both diseases was also studied.

\section{Patients and methods}

PATIENTS

Serum samples were obtained from 100 patients with CD and 101 patients with UC, all unrelated. Diagnosis was based on the usual criteria. ${ }^{1}$ Table 1 summarises their clinical data. Disease activity was assessed using the Crohn's disease activity index (CDAI). ${ }^{13}$ In patients with UC, disease was regarded as quiescent when quiescent or mildly active, or active when moderately active or severe according to the Truelove and Witts index. ${ }^{14}$ Patients who had previously been operated on were held to have active disease if there was clinical, biological, or
Lille, 59037 Lille, France.

Accepted for publication 19 January 1998 
Table 1 Clinical details of patients with Crohn's disease and ulcerative colitis

\begin{tabular}{lll}
\hline & Crohn's disease & Ulcerative colitis \\
\hline No of patients & 100 & 101 \\
M/F & $39 / 61$ & $51 / 50$ \\
Mean age (y) & 30.1 & 40.5 \\
Disease duration (y) & & \\
$\quad<5$ & 60 & 40 \\
$5-10$ & 29 & 23 \\
$>10$ & 11 & 38 \\
Active/quiescent & $47 / 53$ & $28 / 73$ \\
Disease location & & \\
Small bowel & 21 & \\
Colon & 37 & \\
Small bowel and colon & 42 & 8 \\
Distal & & 21 \\
Left & & 19 \\
Pancolic & & 53 \\
Previous colectomy & 21 & 53 \\
Previous surgery & & \\
Medical treatment & & \\
$\quad$ Sulphasalazine or & 26 & 11 \\
$\quad$ mesalazine & 9 & \\
Corticosteroids & 13 & \\
Immunosuppressive & & \\
drugs & 9 & \\
Other treatment & & \\
\hline
\end{tabular}

endoscopic evidence of activity. We also studied sera from 27 patients with other miscellaneous colitides/diarrhoeal illnesses. These patients consisted of seven with collagenous colitis, six with acute self limited colitis, two with eosinophilic colitis, two with chronic radiation proctitis, two with subacute colonic schistosomiasis (Schistosoma haematobium), two with infectious colitis, one with pseudomembranous colitis, one with lymphocytic colitis, one with acute diverticulitis, one with systemic mastocytosis presenting with colonic mucosal involvement, one with coeliac disease, and one with sarcoidosis with colonic involvement. Sera from 163 healthy hospital staff members and blood donors without any history of gastrointestinal disease or familial history of IBD were used as controls.

All serum samples were stored at $-40^{\circ} \mathrm{C}$ until assayed. Investigators had no knowledge of the diagnosis or clinical features at the time serological tests were conducted.

ANCA INDIRECT IMMUNOFLUORESCENCE ASSAY Determination of pANCA was performed by an indirect immunofluorescence technique on ethanol fixed leucocytes according to the first International Workshop on ANCA. ${ }^{15}$ Fluorescein isothiocyanate conjugated rabbit antihuman IgG (specific for $\gamma$ chains) (Dako, Glostrup, Denmark) was used. Patient sera were screened at a dilution of $1 / 20$ in phosphate buffered saline. All slides were assessed by two well trained observers in a blinded fashion.

ASCA ELISA

Antigen consisted of phosphopeptidomannan (PPM) extracted from yeast cells from cultures in bioreactors. ELISA was performed as previously described. ${ }^{12}$ Briefly, plates were coated with $100 \mu \mathrm{l}$ of PPM at a concentration of 1 $\mu \mathrm{g} / \mathrm{ml}$ in sodium carbonate buffer $(60 \mathrm{mM}, \mathrm{pH}$ 9.6), for one hour at $37^{\circ} \mathrm{C}$ and overnight at $4^{\circ} \mathrm{C}$, in moist chambers, and then washed four times in TNT $(50 \mathrm{mM}$ Tris- $\mathrm{HCl}, 150 \mathrm{mM}$ $\mathrm{NaCl}, 0.05 \%$ Tween 20, pH 7.5). Patient sera were diluted $1 / 1000$ in TNT and tested in duplicate. Alkaline phosphatase labelled goat antihuman immunoglobulin (IgG, IgA, IgM; H and L chains; Zymed, Biosoft, Paris, France) was diluted $1 / 3000$ in TNT. A colour reaction was obtained by using substrate Biotrol EIA 405 (Biotrol, Paris, France) for alkaline phosphatase. The plates were read at $405 \mathrm{~nm}$ on an Immunotech (Luminy, France) automatic reader. A coefficient of variation of less than $2 \%$ corresponded to repeatability of optical density values on a single microtitre plate. Each new set of experiments involved sera from four patients exhibiting graded ASCA levels as controls. Interseries reproducibility of ASCA values showed a coefficient of variation of less than $5 \%$.

In our previous study, ${ }^{12}$ we described the standardisation procedure which was designed to avoid variations in individual values observed between series of the immunological assay. Briefly, this involved the use of a standard consisting of a pool of CD patient sera strongly reacting with $S$ cerevisiae mannan. Each set of experiments involved six dilutions of the standard (1/500-1/32 000) from which a standard curve was derived. The highest absorbance (saturation) observed at $405 \mathrm{~nm}$, was arbitrarily defined as $100 \%$ reactivity. Results of individual sera were expressed as a relative reactivity extrapolated from the standard curve and calculated by the ELIOT program (Immunotech, Luminy, France). The upper limit of normality, which gave the best compromise between sensitivity and specificity for CD versus UC, was determined following the establishment of receiver operating characteristics (ROC) curves. Curves were fitted using the SAS program, which indicated for each value the discriminating capacity in sensitivity and specificity. ${ }^{16}$ This was then used to determine the threshold of the test $(3.12 \%)$.

\section{STATISTICAL ANALYSIS}

Sensitivity was defined as the probability of a positive test result in a patient with the disease under investigation. Specificity was defined as the probability of having a negative result in a patient without the disease under investigation. The positive predictive value was defined as the probability of being affected with the disease in a patient with a positive test result. It depends on the prevalence of the disease in the population under investigation and can be determined from a formula based on the Bayes theorem of conditional probability. ${ }^{17}$ The prevalences of CD and UC in Northern France are not known, but the incidences per 100000 population are 4.9 and 3.2, respectively. ${ }^{1}$ To calculate positive predictive values, we took the ratio of incidences of CD and UC to represent the ratio of their prevalences. When studying the relation between test results and clinical parameters, the $\chi^{2}$ test or Fisher's exact test was used when appropriate, and a multivariate analysis was performed using a logistic regression model. Significance was assigned to any probability value less than 0.05 . 
Table 2 Test results for diagnosing either ulcerative colitis or Crohn's disease in patients with inflammatory bowel disease

\begin{tabular}{llllll}
\hline Test & $\begin{array}{l}\text { Ulcerative colitis } \\
(n=101)\end{array}$ & $\begin{array}{l}\text { Crohn's disease } \\
(n=100)\end{array}$ & $\begin{array}{l}\text { Sensitivity } \\
(\%)\end{array}$ & $\begin{array}{l}\text { Specificity } \\
(\%)\end{array}$ & $\begin{array}{l}\text { PPV } \\
(\%)\end{array}$ \\
\hline pANCA+ & 66 & 15 & 65 & 85 & $74^{\star}$ \\
ASCA+ & 12 & 61 & 61 & 88 & $89 \dagger$ \\
pANCA+ASCA- & 58 & 3 & 57 & 97 & $92.5^{\star}$ \\
pANCA-ASCA+ & 3 & 49 & 49 & 97 & $96 \dagger$
\end{tabular}

^For ulcerative colitis; †for Crohn's disease.

PPV, positive predictive value.

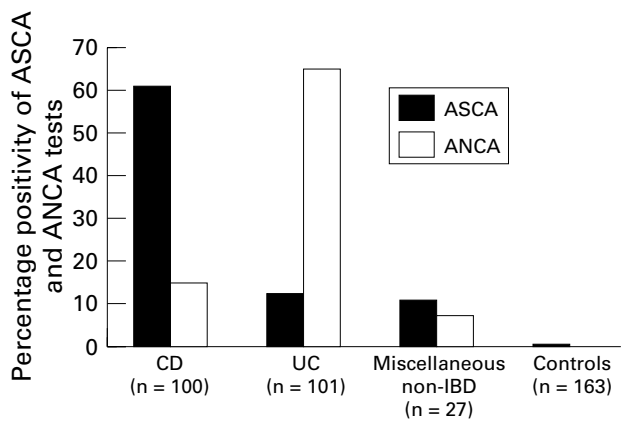

Figure 1 ASCA and ANCA results in the different study groups.

\section{Results}

Figure 1 shows perinuclear ANCA and ASCA results in the different groups. Table 2 shows performances of both tests used either singly or combined in 201 patients with IBD. Among patients with UC, no relation was observed between the presence of pANCA and any clinical parameter including site of disease, disease activity, treatment, or previous surgery (data not shown). Age at diagnosis was significantly less in patients with CD who were ASCA positive than in patients who were negative: 21 years (median), 10-59 (range) versus 24 years $(15-47) \quad(p<0.05)$. Percentages of ASCA positivity were $62 \%, 46 \%$, and $74 \%$ in patients with small bowel involvement, pure colonic disease, and both small bowel and colon involvement, respectively. The prevalence of ASCA was significantly higher in patients with small bowel involvement (with or without colonic disease) than in patients with pure colonic disease: $70 \%$ versus $46 \%$ $(p<0.05)$. The presence of ASCA was independent of disease activity and treatment. In the multivariate analysis, only small bowel location was significantly associated with ASCA positivity (adjusted odds ratio $2.7 ; 95 \%$ confidence interval 1.1-6.7; $\mathrm{p}=0.027$ ). Patients with CD who were pANCA positive had no particular clinical features compared with patients who were pANCA negative. Patients with UC who were ASCA positive had no particular clinical features compared with patients who were ASCA negative.

Among 27 patients with miscellaneous nonIBD disorders, one with eosinophilic colitis was ASCA positive and pANCA positive, two were ASCA positive and pANCA negative (one with radiation proctitis, one with sarcoidosis) and one was ASCA negative and pANCA positive (collagenous colitis). One the 163 controls was ASCA positive.

\section{Discussion}

In the present study, we assessed the prevalence of ASCA and pANCA in a large population and their value for differentiating between UC and CD and between IBD and other colitides. At present, it appears that ASCA and pANCA are strongly associated with CD and UC, respectively.

Our percentages of serum samples from patients with UC and CD which were pANCA positive are comparable to the data reported in Western Europe. ${ }^{18}$ Overall sensitivity (61\%) and specificity (88\%) of ASCA for CD in this study were similar to those reported in a smaller series of adult patients, ${ }^{12}$ and more recently in a paediatric series. ${ }^{19}$ After combining the two tests, sensitivity dropped by approximately $10 \%$, as would be expected, but specificity increased to more than $95 \%$ yielding a very high positive predictive value for this combination. Among patients with miscellaneous non-IBD disorders, only three were ASCA positive and two were pANCA positive. These tests could thus be useful in clinical practice to differentiate between IBD and other colitides. The clinical relevance of serological tests also relates to differentiation of CD from UC in patients with colitis. Combination of both tests yielded high positive predictive values for the diagnosis of either UC or CD, which could make them usefully applicable to individual patients. In our study population of 37 patients with pure colonic $\mathrm{CD}$, sensitivity dropped to $45 \%$ for ASCA and to $32 \%$ for the association pANCA negative/ASCA positive (12/37). However, the high positive predictive value would allow an accurate diagnosis in one third of these patients.

Increasing evidence supports the concept of clinical and genetic heterogeneity in IBD and serum immune markers have been used to characterise subgroups of patients. ${ }^{20}$ The presence of pANCA in UC was independent of disease activity and extent; pANCA were also present in patients who had been operated on, confirming previous reports..$^{3-82122}$ Vasiliauskas et al reported that $100 \%$ of patients with CD who were pANCA positive had "UC like features". ${ }^{23}$ More recently the same group reported that there was a negative correlation between mean ANCA level and ASCA expression and that $100 \%$ of DNAse sensitive pANCA/ASCA negative patients with CD had the "UC like" phenotype. ${ }^{24}$ This suggests that a phenotypically distinct group of CD could be defined by their pANCA and ASCA status. In the present study, the presence of pANCA could not be associated with particular clinical features, thus confirming our previous report. ${ }^{25}$ However, the techniques used to identify ANCA were different in Vasiliauskas et al's study and ours. This further underscores the need for international standardisation for ANCA determination in IBD. ${ }^{25} 26$

Patients with CD who were ASCA positive were younger than those who were ASCA negative, and the prevalence of ASCA was significantly associated with small bowel involvement. Using multivariate analysis, only small bowel involvement was significantly 
associated with ASCA positivity. However, age and disease location are correlated: in this series, patients with small bowel involvement were younger at diagnosis than patients with pure colonic disease. The literature supports the concept of more small bowel disease in childhood and adolescent series and more colonic disease in older onset populations. ${ }^{27} 28$ Other series are needed to confirm whether ASCA represent a serological marker of a clinical subgroup with younger age at diagnosis and more frequent small bowel involvement.

The present study has shown that the combined use of ASCA and pANCA could differentiate CD from UC and other colitides although the relatively low prevalence of ASCA in patients with CD colitis may limit its clinical usefulness. Assaying for these markers may reveal clinical subgroups. However, since various prevalences of serological markers, such as pANCA, have been observed worldwide, ${ }^{5-8}$ it is recommended that multicentre, prospective studies be conducted.

This work was supported by a grant from the Institut de Recherche des Maladies de l'Appareil Digestif (IRMAD), the INSERM (CRI 4UOO4B), and the Centre Hospitalier et Universitaire de Lille (Contrat 96/38/9713)

1 Gower-Rousseau C, Salomez JL, Dupas JL, et al. Incidence of inflammatory bowel disease in northern France (1988-1990). Gut 1994;35:1433-8.

2 Sandler RS. Epidemiology of inflammatory bowel disease. In: Targan SR, Shanahan F, eds. Inflammatory bowel disease. From bench to bedside. Baltimore: Williams and Wilkins, 1993:5-30.

3 Saxon A, Shanahan F, Landers C, et al. A distinct subset of antineutrophil cytoplasmic antibodies is associated with inflammatory bowel disease. 7 Allergy Clin Immunol 1990;86:202-10.

4 Rump JA, Schölmerich J, Gross V, et al. A new type of perinuclear antineutrophil cytoplasmic antibody (p-ANCA) in active ulcerative colitis but not in Crohn's disease. Immunobiology 1990;181:406-13.

5 Duerr RH, Targan SR, Landers CJ, et al. Anti-neutrophil cytoplasmic antibodies in ulcerative colitis: comparison with other colitides/diarrheal illnesses. Gastroenterology with other colitide

6 Colombel JF, Reumaux D, Duthilleul P, et al. Antineutrophil cytoplasmic autoantibodies in inflammatory bowel discytoplasmic autoantibodies in inflammatory
eases. Gastroenterol Clin Biol 1992;16:656-60.

7 Cambridge G, Rampton DS, Stevens TRJ, et al. Antineutrophil antibodies in inflammatory bowel disease: prevalence and diagnostic role. Gut 1992;33:668-74.

8 Oudkerk Pool M, Ellerbroek PM, Ridwan BU, et al. Serum antineutrophil cytoplasmic autoantibodies in inflammatory bowel disease are mainly associated with ulcerative colitis. A correlation study between perinuclear antineutrophil cytoplasmic autoantibodies and clinical parameters, medical, and surgical treatment. Gut 1993;4:46-50.

9 Main J, McKenzie H, Yeaman GR, et al. Antibody to Saccharomyces cerevisiae (baker's yeast) in Crohn's disease. BMF 1988;297:1105-6.

10 Barnes RMR, Allan S, Taylor-Robinson $\mathrm{CH}$, et al. Serum antibodies reactive with Saccharomyces cerevisiae in inflammatory bowel disease. Is IgA antibody a marker for Crohn's disease? Int Arch Allergy Appl Immunol 1990;92:915.

11 Giaffer $\mathrm{MH}$, Clark A, Holdsworth CD. Antibodies to Saccharomyces cerevisiae in patients with Crohn's disease and their possible pathogenic importance. Gut 1992;33: $1071-5$.

12 Sendid B, Colombel JF, Jacquinot PM, et al. Specific antibody response to oligomannosidic epitopes in Crohn's disease. Clin Diag Lab Immunol 1996;3:219-26.

13 Best WR, Becktel JM, Singleton JW. Rederived values of the eight coefficients of the Crohn's disease activity index. Gastroenterology 1979;77:843-6.

14 Truelove SC, Witts LJ. Cortisone in ulcerative colitis. Final report on a therapeutic trial. BMf 1955;2:1041-8.

15 Wiik A. Delineation of a standard procedure for immunofluorescence detection of ANCA. APMIS 1989;97(suppl 1):12-13.

16 Campbell G. Advances in statistical methodology for the evaluation of diagnostic and laboratory tests. Stat Med 1994;13:499-508.

17 Grenier B. Décision médicale, 1990:59-68.

18 Oudkerk Pool M, Roca M, Reumaux D, et al. The value of pANCA as a serological marker for ulcerative colitis in different European regions. Eur 7 Gastroenterol Hepatol 1994. 6:399-403.

19 Seidman EG, Ruemmele FM, Landers G, et al. Disease specificity diagnostic accuracy of new serological tests in pediatric IBD [abstract]. Gastroenterology 1997;112:A1087.

20 Yang H, Rotter JL. Genetics of inflammatory bowel disease. In: Targan SR, Shanahan F, eds. Inflammatory bowel disease. From bench to beside. Baltimore: Williams and Wilkins, 1994:32-64.

21 Reumaux D, Colombel JF, Delecourt L, et al. Antineutrophil cytoplasmic auto-antibodies (ANCA) in patients with ulcerative colitis: influence of disease activity and familial study. Adv Exp Med Biol 1993;336:515-18.

22 Reumaux D, Colombel JF, Duclos B, et al, and GETAID. Antineutrophil cytoplasmic auto-antibodies (ANCA) in sera with ulcerative colitis after proctocolectomy with ileoanal anastomosis. Adv Exp Med Biol 1993;336:523-5.

23 Vasiliauskas EA, Plevy SE, Landers CJ, et al. Perinuclear Vasiliauskas EA, Plevy SE, Landers CJ, et al. Perinuclear
antineutrophil cytoplasmic antibodies in patients with Crohn's disease define a clinical subgroup. Gastroenterology 1996;110:1810-19.

24 Vasiliauskas EA, Plevy SE, Targan SR. Stratification of Crohn's disease by antineutrophil cytoplasmic antibodies (ANCA) and anti-Saccharomyces cerevisiae antibody (ASCA) distinguishes phenotypic subgroups [abstract]. Gastroenterology 1997;112:A1112.

25 Jamar-Leclerc N, Reumaux D, Duthilleul P, et al. Do pANCA define a clinical subgroup in patients with Crohn's disease [letter]? Gastroenterology 1997;112:316.

26 Vasiliauskas EA, Targan SR. Do pANCA define a clinical subgroup in patients with Crohn's disease [reply]? subgroup in patients with Cro

27 Polito JM II, Childs B, Mellits ED, et al. Crohn's disease: influence of age at diagnosis on site and clinical type of disease. Gastroenterology 1996;111:580-6.

28 Colombel JF, Grandbastien B, Gower-Rousseau C, et al. Clinical characteristics of Crohn's disease in 72 families. Gastroenterology 1996;111:604-7. 